\title{
AVALIAÇÃO DA ADERÊNCIA DE ARGAMASSA EM BLOCOS CERÂMICOS
}

\author{
NUNES, IGO HENRIQUE SILVA \\ Engenheiro Civil \\ Unipar \\ PR; BR \\ igo@prof.unipar.br
}

\author{
HARTMANN, VICTOR MOURÃO \\ Engenheiro Civil \\ Unipar \\ PR; BR \\ victor.h@edu.unipar.br
}

\author{
SILVA, ANTONIO AUGUSTO QUITINO DA \\ Engenheiro Civil \\ Unipar \\ PR; BR \\ antonio.quintino@edu.unipar.br
}

\section{RESUMO}

O objetivo deste trabalho foi verificar e avaliar a influência do aditivo adesivo Bianco ${ }^{\circledR}$ da empresa Vedacit ${ }^{\circledR}$ na aderência e permeabilidade da argamassa aplicada no substrato. Para a realização dos ensaios foram utilizados tijolos cerâmicos com chapisco. O chapisco foi desenvolvido com traço 1:3 em volume, e para a confecção das argamassas, foi empregado o traço 1:1:6 (cimento:cal:areia) em volume e o mesmo traço com adição do aditivo. No estado fresco foram realizados os ensaios de

densidade, teor de ar e consistência da argamassa onde se notou que a argamassa com aditivo apresentou 2\% de melhora no índice de consistência em relação à argamassa convencional. Após 28 dias foram realizados no estado endurecido os ensaios de permeabilidade onde a argamassa com aditivo adesivo apresentou melhor índice, tornando-se menos permeável e no ensaio de resistência de aderência à tração proporcionou uma melhora de $14 \%$ no desempenho da aderência em relação à argamassa convencional.

Palavras-chave: argamassa, aderência, revestimento.

\section{ABSTRACT}

The objective of this work was evaluate the influence of the Bianco ${ }^{\circledR}$ adhesive additive fromVedacit ${ }^{\circledR}$ on adhesion and permeability of the mortar applied to the substrate. For the realization of the tests were used ceramic bricks with slurry mortars. The slab was developed with a 1: 3 trace by volume, and the 1: 1: 6 (cement: lime: sand) trace in the volume and the same trait with

addition of additive were used for the preparation of the mortars. In the fresh state, the tests of density, air content and mortar consistency were performed, in which the mortar with additive showed a $2 \%$ improvement in the consistency index compared to conventional mortar. After 28 days were performed in the hardened state the permeability tests where the mortar with adhesive additive presented better index, becoming less permeable and in the test of resistance to adhesion

to the traction provided a $14 \%$ improvement in the performance of the adhesion in relation to the conventional mortar. Keywords: mortar, adherence, coating

\section{INSTRUÇÕES GERAIS}

As argamassas possuem diversas finalidades, como por exemplo, assentamento de tijolos, blocos, azulejos e regularização da superfície de substratos. As argamassas são empregadas durante a execução de uma obra, desde o canteiro inicial até a conclusão da mesma (GOMES; NEVES 2002).

A proteção que as argamassas proporcionam ao substrato constitui parcela essencial na eficiência e durabilidade das edificações, podendo-se dizer que as mesmas são a pele das construções. A argamassa tem a incumbência de proteção contra a exposição ao tempo, quando aplicada no exterior da edificação; vedação que contribui de forma coletiva a favor do isolamento térmico, isolamento acústico, estanqueidade, segurança contra incêndio e resistência ao desgaste de abalos superficiais; e também para regularização da superfície a fins de assentamento do revestimento cerâmico (CARASEK, 2007). 
O desempenho da aderência das argamassas nos substratos depende de fatores como a proporção dos materiais que a constitui, de suas características e do cuidado no armazenamento desses materiais (GOMES; NEVES 2002). Assim, a capacidade dá argamassa de atingir uma completa, resistente e durável aderência com a base, talvez seja á mais importante propriedade concernente ao comportamento de um revestimento (IOPPI, 1995). A argamassa de boa qualidade deve conter propriedades mecânicas, uma combinação que depende da característica dos materiais aliado ao preparo e manuseio dos mesmos (SANTOS, 2008). A falta de aderência pode ser influenciada, muitas vezes, por não atender a NBR 13528 (ABNT, 2010), pela falta de conhecimento técnico, ou por materiais de baixa qualidade. Uma forma de melhorar o desempenho de aderência da argamassa aplicada ao substrato é com o chapisco, que é um tratamento prévio do substrato, criando uma rugosidade na base e regularizando a absorção inicial. Normalmente, a falta e a perda de desempenho do revestimento podem causar prejuízos econômicos, afetando a edificação em conforto, estética e a sua integridade (CARASEK, 1996).

Este trabalho tem como objetivo avaliar a influência no emprego do aditivo adesivo na argamassa aplicada no substrato, seguindo as metodologias das normas brasileiras, tais como a NBR 13276 (ABNT 2005), NBR 13278 (ABNT, 2005), NBR 13528 (ABNT, 2010) e CSTC (1982). O modelo baseia-se nas características e traços comuns existentes nas obras da construção civil no território nacional. O trabalho mescla um estudo de literatura sobre a avaliação e o desempenho da aderência dos revestimentos de argamassa, objetivando o desempenho na aderência das argamassas nos substratos com a idade de 28 dias, utilizando como métodos de ensaios, o de permeabilidade pelo método do cachimbo, determinação da consistência e da resistência à tração.

\section{MATERIAIS E MÉTODOS}

O desenvolvimento da pesquisa foi elaborado de acordo com o fluxograma apresentado na Figura 1

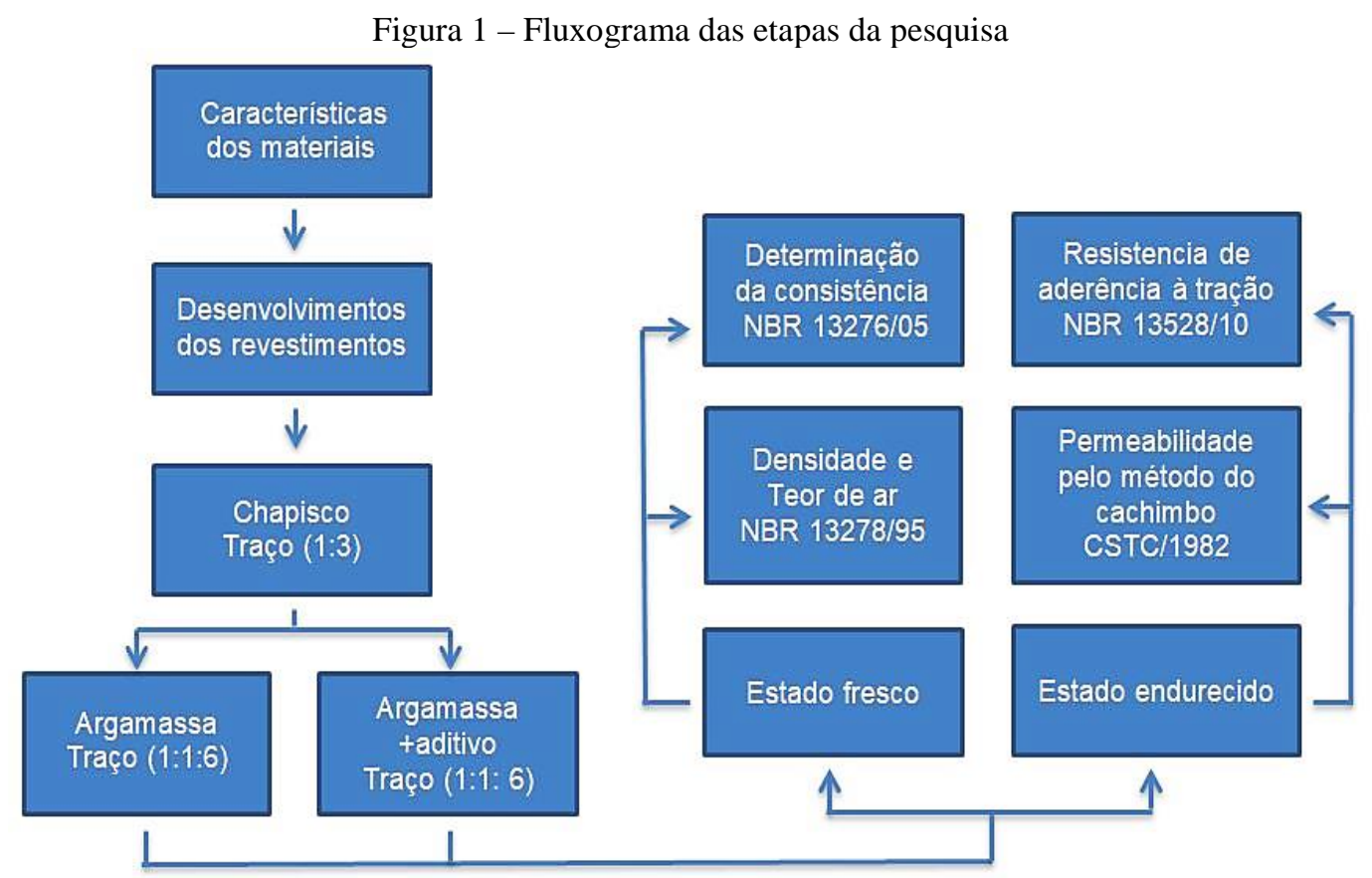

Os traços apresentados no fluxograma estão em volume e as características físicas dos materiais utilizados para a composição das argamassas estão apresentados na Tabela 1.

Tabela 1 - Características dos materiais

\begin{tabular}{c|c|c|c|c|c|c|c}
\hline \multirow{2}{*}{ Característica } & \multicolumn{2}{|c|}{ Areia } & \multicolumn{2}{c|}{ Cal } & \multicolumn{2}{c|}{ Cimento } & $\begin{array}{c}\text { Aditivo } \\
\text { adesivo }\end{array}$ \\
\hline Tipo & \multicolumn{2}{|c|}{ Natural } & \multicolumn{2}{c|}{ Hidratada CH-III } & CPII E-32 & $\begin{array}{c}\text { Bianco } \\
\text { Vedacit }^{\circledR}\end{array}$ \\
\hline Massa unitária $\left(\mathrm{g} / \mathrm{cm}^{\circledR}\right)$ & $\begin{array}{c}\text { NBR 7251 } \\
(\text { ABNT, 1982) }\end{array}$ & 1,4 & --- & --- & --- & --- & --- \\
\hline $\begin{array}{c}\text { Massa especifica real } \\
\left(\mathrm{g} / \mathrm{cm}^{3}\right)\end{array}$ & $\begin{array}{c}\text { NBR 9776 } \\
(\text { ABNT, 1987) }\end{array}$ & 2,6 & $\begin{array}{c}\text { NBR 6474 } \\
\text { (ABNT, }\end{array}$ & 2,3 & $\begin{array}{c}\text { NBR 6474 } \\
\text { (ABNT, }\end{array}$ & 3,1 & 1,02 \\
\hline
\end{tabular}




\begin{tabular}{c|c|c|c|c|c|c|c}
\hline & & & $2001)$ & & $2001)$ & & \\
\hline Módulo de finura & $\begin{array}{c}\text { NBR 7217 } \\
\text { (ABNT, 1987) }\end{array}$ & 4,1 & --- & --- & --- & --- & --- \\
\hline $\begin{array}{c}\text { Teor de finos }<0,0075 \mathrm{~mm} \\
(\%)\end{array}$ & $\begin{array}{c}\text { NBR NM 46 } \\
\text { (ABNT, 2003) }\end{array}$ & 0,71 & $\begin{array}{c}\text { NBR 7175 } \\
\text { (ABNT, } \\
2003)\end{array}$ & 95 & $\begin{array}{c}\text { NBR NM } \\
\text { (ABNT, } \\
2003)\end{array}$ & 87 & --- \\
\hline
\end{tabular}

O chapisco utilizado foi desenvolvido com a dosagem em volume de 1:3 (cimento: areia) estabelecida por Zanelato (2015), e aplicado sobre a superfície do substrato cerâmico com ajuda de um aplicador manual de chapisco, para melhorar a rugosidade.

Foram desenvolvidos dois tipos de argamassas, uma argamassa mista de cimento, cal e areia, em volume (1:1:6) estabelecida de acordo com Zanelato (2015), e outra de traço igual, porém com aditivo adesivo Bianco ${ }^{\circledR}$ da Vedacit ${ }^{\circledR}$, onde foi utilizado 100ml para cada 2 litros de água. No estado fresco as argamassas foram submetidas aos respectivos ensaios: determinação da consistência, de acordo com a NBR 13276 (ABNT, 2005); densidade e teor de ar incorporado, de acordo com a NBR 13278 (ABNT, 2005).

Para o estudo das argamassas no estado endurecido, estas foram aplicadas seguindo o modelo da Tabela 2.

Tabela 2 - Programa experimental

\begin{tabular}{c|c|c|c}
\hline Substrato & Argamassas & Preparo da base & Condições da base \\
\hline \multirow{2}{*}{ Tijolo cerâmico } & Convencional & Com chapisco & Superfície molhada \\
\cline { 2 - 4 } & Convencional + aditivo adesivo & Com chapisco & Superfície molhada \\
\hline
\end{tabular}

Foram aplicadas sobre tijolos cerâmicos de 6 furos $(9 \times 14 \times 19 \mathrm{~cm})$ como substrato. Os tijolos foram adquiridos no comércio do município de Juranda-PR. Após serem limpos, os tijolos foram molhados e em seguida aplicou-se o chapisco sobre os mesmos e aguardado 72 horas de cura do chapisco, conforme recomendado pela NBR 7200 (ABNT, 1998). Para aplicação da argamassa sobre o chapisco, e padronização da espessura, foi criado um gabarito de madeira para revestir os tijolos; o tijolo ficou 1,5cm abaixo da borda superior do gabarito, onde, com a colher de pedreiro, foram aplicadas as argamassas, pressionando-as para aumentar a compacidade, como ilustrado nas Figuras 11, 12 e 13. A argamassa ficou com uma espessura de 1,5cm (parede interna).

Foram utilizados 28 tijolos, sendo 4 tijolos para o ensaio de permeabilidade pelo método do cachimbo e 24 tijolos para ensaio de resistência de aderência à tração. Foram realizados 28 dias de cura da argamassa, e após a realização dos ensaios de permeabilidade e aderência.

\subsection{Permeabilidade pelo método do cachimbo}

Para o ensaio de permeabilidade, foram seguidas as especificações do CSTC (1982). Foram adaptados seringas de $20 \mathrm{ml}$ como cachimbo. O ensaio foi realizado após os 28 dias de cura da argamassa. Com a ajuda de uma massa plástica para calafetar, os cachimbos foram colados no centro do tijolo na posição vertical, e foram cheios com água. A leitura do ensaio foi realizada com os tempos de 5, 10 e 15 minutos. No total foram realizados 4 ensaios, sendo 2 ensaios para cada argamassa. Foram adaptados seringas de $20 \mathrm{ml}$ como cachimbo, devido à indisponibilidade dos materiais.

\subsection{Resistência de aderência a tração}

O ensaio de resistência de aderência à tração foi realizado de acordo com a NBR 13528 (ABNT, 2010) após os 28 dias de cura da argamassa. Com a ajuda de uma serra copo diamantada $53 \mathrm{~mm}$ de diâmetro, foram realizados dois cortes em cada tijolo, corte A e B, até atingir uma profundidade de 3 a 5 milímetros, totalizando 24 cortes para argamassa convencional e 24 para argamassa com aditivo adesivo. Posteriormente, foram coladas as pastilhas com uma solda plástica no centro das áreas demarcadas com os cortes; logo após a secagem da cola (verificada de acordo com o fabricante), foram aplicadas as cargas com o auxilio de um dinamômetro. Os valores registrados e as superfícies, onde ocorreu à ruptura, foram anotados. 


\section{RESULTADOS}

Os resultados obtidos dos ensaios apontados no fluxograma são apresentados a seguir. Inicialmente são exibidos os resultados da caracterização das argamassas, ou seja, dos ensaios no estado fresco e, em seguida, dos ensaios no estado endurecido.

\subsection{Caracterização da argamassa no estado fresco}

3.1.1 Determinação da consistência - método de ensaio NBR 13276 (ABNT, 2005)

Os resultados estão sendo apresentados na Tabela 3. As quantidades de água utilizadas foram adotadas pelos autores para adiquirir índices de consistência que estivessem dentro do intervalo da norma, 255 a 265mm.

Tabela 3 - Índice de consistência da argamassa

\begin{tabular}{c|c|c|c}
\hline Argamassas & Tentativas & Quantidade de água (g) & Índice de consistência (mm) \\
\hline Convencional & $1^{\mathrm{a}}$ & 330 & 295 \\
\hline Convencional & $2^{\mathrm{a}}$ & 290 & 262 \\
\hline Com aditivo adesivo & $1^{\mathrm{a}}$ & 300 & 317 \\
\hline Com aditivo adesivo & $2^{\mathrm{a}}$ & 280 & 259 \\
\hline
\end{tabular}

A partir dos resultados atingidos, aponta-se que a argamassa convencional necessita de 290g de água para 1,5Kg de argamassa, já a argamassa com aditivo adesivo necessita de $280 \mathrm{~g}$ de água para $1,5 \mathrm{Kg}$ de argamassa com o intuito de assegurar uma boa trabalhabilidade durante os ensaios.

\subsubsection{Densidade e teor de ar - método de ensaio NBR 13278 (ABNT, 2005)}

Na Tabela 4 apresentaram-se os valores obtidos através dos ensaios. A norma não especifica parâmetros ou limites para estes ensaios.

Tabela 4 - Densidade e teor de ar das argamassas

\begin{tabular}{c|c|c}
\hline Argamassas & Densidade $\left(\mathrm{Kg} / \mathrm{m}^{3}\right)$ & Teor de $\mathrm{ar}(\%)$ \\
\hline Convencional & 1867 & 15,9 \\
\hline Com aditivo adesivo & 1937 & 16,5 \\
\hline
\end{tabular}

De acordo com os resultados, a argamassa com aditivo adesivo possui maior teor de ar incorporado, indicando que possui uma melhoria nas suas propriedades no estado fresco. A aumento do teor de ar pode afetar a aderência na interface da argamassa no estado endurecido, por possuir muitos espaços vazios na argamassa.

\subsection{Caracterização da argamassa no estado endurecido}

A Tabela 5 apresenta os resultados obtidos através do trabalho experimental realizado.

Tabela 5 - Leituras do ensaio de permeabilidade

\begin{tabular}{c|c|c|c|c}
\hline \multirow{2}{*}{ Argamassa } & \multirow{2}{*}{ Tentativa } & \multicolumn{3}{|c}{ Absorção (ml) } \\
\cline { 3 - 5 } & & $5 \min$ & $10 \mathrm{~min}$ & $15 \mathrm{~min}$ \\
\hline Convencional & $1^{\mathrm{a}}$ & 3 & 5,5 & 8 \\
\hline Convencional & $2^{\mathrm{a}}$ & 6 & 10 & 14 \\
\hline Com aditivo adesivo & $1^{\mathrm{a}}$ & 4 & 6,5 & 9 \\
\hline Com aditivo adesivo & $2^{\mathrm{a}}$ & 4 & 7 & 9 \\
\hline
\end{tabular}

Observou-se que conforme passavam os minutos, ambas as argamassas foram aumentando a absorção. Observando o comportamento da curva, nota-se que a argamassa convencional absorve mais água com o decorrer do tempo em relação à argamassa com aditivo adesivo, destacando que o aditivo empregado na argamassa possui características impermeabilizantes. 
Os resultados obtidos após o tempo determinado e para as combinações propostas estão apresentados na Tabela 6, 7, 8 e 9.

Tabela 6 - Resultados individuais para argamassa convencional (corte A)

\begin{tabular}{|c|c|c|c|c|c|c|c|c|c|c|c|}
\hline \multicolumn{2}{|c|}{ Corpo de prova } & \multirow[b]{2}{*}{ Carga de ruptura (N) } & \multirow[b]{2}{*}{ Tensão Ra (MPa) } & \multicolumn{8}{|c|}{ Forma de ruptura (\%) } \\
\hline $\mathrm{N}^{\mathrm{o}}$ & Área $\left(\mathrm{mm}^{2}\right)$ & & & 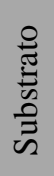 & 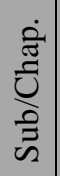 & 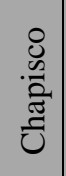 & 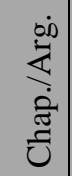 & 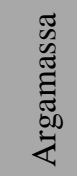 & $\begin{array}{c}\frac{\pi}{0} \\
\stackrel{0}{0} \\
\dot{000} \\
\dot{4}\end{array}$ & $\frac{\pi}{0}$ & 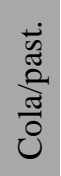 \\
\hline $1 \mathrm{~A}$ & 1963,50 & 173 & 0,088 & & & & 75 & 25 & & & \\
\hline $2 \mathrm{~A}$ & 1963,50 & 172 & 0,087 & & & 95 & & 5 & & & \\
\hline $3 \mathrm{~A}$ & 1963,50 & 77 & 0,039 & & & 5 & & 95 & & & \\
\hline $4 \mathrm{~A}$ & 1963,50 & 265 & 0,134 & & & & 90 & 10 & & & \\
\hline $5 \mathrm{~A}$ & 1963,50 & 150 & 0,076 & & & & & 100 & & & \\
\hline $6 \mathrm{~A}$ & 1963,50 & 394 & 0,200 & & & & & 100 & & & \\
\hline $7 \mathrm{~A}$ & 1963,50 & 307 & 0,156 & & & & & 100 & & & \\
\hline $8 \mathrm{~A}$ & 1963,50 & 265 & 0,134 & & & & 85 & 15 & & & \\
\hline $9 \mathrm{~A}$ & 1963,50 & 306 & 0,155 & & & & & 100 & & & \\
\hline $10 \mathrm{~A}$ & 1963,50 & 182 & 0,092 & & & & & 100 & & & \\
\hline $11 \mathrm{~A}$ & 1963,50 & 273 & 0,139 & & & & & 100 & & & \\
\hline $12 \mathrm{~A}$ & 1963,50 & 160 & 0,081 & & & & & 100 & & & \\
\hline \multicolumn{3}{|c|}{ Média (MPa) } & \multicolumn{9}{|c|}{0,115} \\
\hline \multicolumn{3}{|c|}{ Desvio Padrão (MPa) } & \multicolumn{9}{|c|}{0,044} \\
\hline \multicolumn{3}{|c|}{ C.V. $(\%)$} & \multicolumn{9}{|c|}{39} \\
\hline
\end{tabular}

Tabela 7 - Resultados individuais para argamassa convencional (corte B)

\begin{tabular}{|c|c|c|c|c|c|c|c|c|c|c|c|}
\hline \multicolumn{2}{|c|}{ Corpo de prova } & \multirow[b]{2}{*}{ Carga de ruptura $(\mathrm{N})$} & \multirow[b]{2}{*}{ Tensão Ra (MPa) } & \multicolumn{8}{|c|}{ Forma de ruptura (\%) } \\
\hline $\mathrm{N}^{\mathrm{o}}$ & Área $\left(\mathrm{mm}^{2}\right)$ & & & 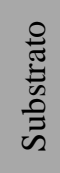 & $\begin{array}{l}\dot{\text { चे }} \\
\text { U্ } \\
\text { की }\end{array}$ & 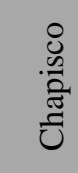 & 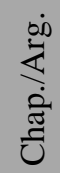 & 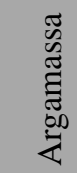 & $\begin{array}{l}\frac{\pi}{0} \\
\dot{e} \\
\dot{0} 0 \\
\end{array}$ & $\frac{\pi}{0}$ & $\begin{array}{l}\dot{0} \\
\tilde{0} \\
\frac{\tilde{d}}{0} \\
0\end{array}$ \\
\hline $1 \mathrm{~B}$ & 1963,50 & 176 & 0,089 & & & 100 & & & & & \\
\hline $2 \mathrm{~B}$ & 1963,50 & 165 & 0,084 & & & 10 & & 90 & & & \\
\hline $3 \mathrm{~B}$ & 1963,50 & 57 & 0,029 & & & 10 & & 90 & & & \\
\hline $4 \mathrm{~B}$ & 1963,50 & 83 & 0,042 & & & 10 & & 90 & & & \\
\hline $5 \mathrm{~B}$ & 1963,50 & 170 & 0,086 & & & & & 100 & & & \\
\hline $6 \mathrm{~B}$ & 1963,50 & 243 & 0,123 & & & & & 100 & & & \\
\hline $7 \mathrm{~B}$ & 1963,50 & 62 & 0,031 & & & 90 & & 10 & & & \\
\hline $8 \mathrm{~B}$ & 1963,50 & 304 & 0,154 & & & 85 & & 15 & & & \\
\hline $9 \mathrm{~B}$ & 1963,50 & 38 & 0,019 & & & 90 & & 10 & & & \\
\hline $10 \mathrm{~B}$ & 1963,50 & 233 & 0,118 & & & & & 100 & & & \\
\hline $11 \mathrm{~B}$ & 1963,50 & 130 & 0,066 & & & & & 100 & & & \\
\hline $12 \mathrm{~B}$ & 1963,50 & 186 & 0,094 & & & 95 & & 5 & & & \\
\hline \multicolumn{3}{|c|}{ Média (MPa) } & \multicolumn{9}{|c|}{0,078} \\
\hline \multicolumn{3}{|c|}{ Desvio Padrão (MPa) } & \multicolumn{9}{|c|}{0,042} \\
\hline \multicolumn{3}{|c|}{ C.V. (\%) } & \multicolumn{9}{|c|}{53,91} \\
\hline
\end{tabular}


Tabela 8 - Resultados individuais para argamassa com aditivo adesivo (corte A)

\begin{tabular}{|c|c|c|c|c|c|c|c|c|c|c|c|}
\hline \multicolumn{2}{|c|}{ Corpo de prova } & \multirow[b]{2}{*}{ Carga de ruptura (N) } & \multirow[b]{2}{*}{ Tensão Ra (MPa) } & \multicolumn{8}{|c|}{ Forma de ruptura (\%) } \\
\hline $\mathrm{N}^{\circ}$ & Área $\left(\mathrm{mm}^{2}\right)$ & & & 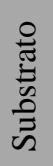 & $\begin{array}{l}\dot{\text { चे }} \\
\text { U } \\
\text { के }\end{array}$ & 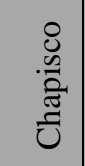 & 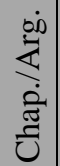 & 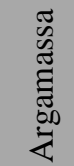 & 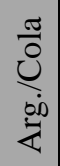 & $\frac{\pi}{0}$ & 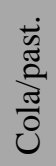 \\
\hline $1 \mathrm{~A}$ & 1963,50 & 153 & 0,077 & & & 90 & & 10 & & & \\
\hline $2 \mathrm{~A}$ & 1963,50 & 238 & 0,121 & & & & & 100 & & & \\
\hline $3 \mathrm{~A}$ & 1963,50 & 318 & 0,161 & & & & & 100 & & & \\
\hline $4 \mathrm{~A}$ & 1963,50 & 271 & 0,138 & & & 90 & & 10 & & & \\
\hline $5 \mathrm{~A}$ & 1963,50 & 417 & 0,212 & & 100 & & & & & & \\
\hline $6 \mathrm{~A}$ & 1963,50 & 250 & 0,127 & & & & & 100 & & & \\
\hline $7 \mathrm{~A}$ & 1963,50 & 160 & 0,081 & & & 10 & & 90 & & & \\
\hline $8 \mathrm{~A}$ & 1963,50 & 123 & 0,062 & & & 100 & & & & & \\
\hline $9 \mathrm{~A}$ & 1963,50 & 171 & 0,087 & & & & & 100 & & & \\
\hline $10 \mathrm{~A}$ & 1963,50 & 151 & 0,076 & & & & & 100 & & & \\
\hline $11 \mathrm{~A}$ & 1963,50 & 231 & 0,117 & & & 5 & & 95 & & & \\
\hline $12 \mathrm{~A}$ & 1963,50 & 220 & 0,112 & & & 95 & & 5 & & & \\
\hline \multicolumn{3}{|c|}{ Média (MPa) } & \multicolumn{9}{|c|}{0,115} \\
\hline \multicolumn{3}{|c|}{ Desvio Padrão (MPa) } & \multicolumn{9}{|c|}{0,043} \\
\hline \multicolumn{3}{|c|}{ C.V. (\%) } & \multicolumn{9}{|c|}{37,21} \\
\hline
\end{tabular}

Tabela 9 - Resultados individuais para argamassa com aditivo adesivo (corte B)

\begin{tabular}{|c|c|c|c|c|c|c|c|c|c|c|c|}
\hline \multicolumn{2}{|c|}{ Corpo de prova } & \multirow[b]{2}{*}{ Carga de ruptura (N) } & \multirow[b]{2}{*}{ Tensão Ra (MPa) } & \multicolumn{8}{|c|}{ Forma de ruptura (\%) } \\
\hline $\mathrm{N}^{\circ}$ & Área $\left(\mathrm{mm}^{2}\right)$ & & & 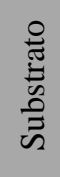 & 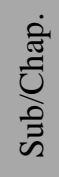 & 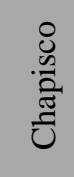 & 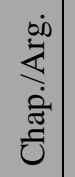 & 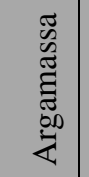 & 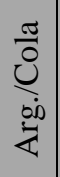 & $\frac{\pi}{0}$ & 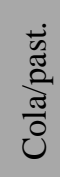 \\
\hline $1 \mathrm{~B}$ & 1963,50 & 147 & 0,07 & & & 95 & & 5 & & & \\
\hline $2 \mathrm{~B}$ & 1963,50 & 211 & 0,107 & & & 95 & & 5 & & & \\
\hline $3 \mathrm{~B}$ & 1963,50 & 276 & 0,140 & & & & & 100 & & & \\
\hline $4 \mathrm{~B}$ & 1963,50 & 191 & 0,097 & & & 95 & & 5 & & & \\
\hline $5 \mathrm{~B}$ & 1963,50 & 274 & 0,140 & & & & & 100 & & & \\
\hline $6 \mathrm{~B}$ & 1963,50 & 300 & 0,153 & & & & & 100 & & & \\
\hline $7 \mathrm{~B}$ & 1963,50 & 280 & 0,143 & & & & & 100 & & & \\
\hline $8 \mathrm{~B}$ & 1963,50 & 115 & 0,058 & & & 100 & & & & & \\
\hline $9 \mathrm{~B}$ & 1963,50 & 190 & 0,097 & & & 15 & & 85 & & & \\
\hline $10 \mathrm{~B}$ & 1963,50 & 70 & 0,035 & & & & & 100 & & & \\
\hline $11 \mathrm{~B}$ & 1963,50 & 301 & 0,153 & & & & & 100 & & & \\
\hline $12 \mathrm{~B}$ & 1963,50 & 214 & 0,109 & & & 90 & & 10 & & & \\
\hline \multicolumn{3}{|c|}{ Média (MPa) } & \multicolumn{9}{|c|}{0,1085} \\
\hline \multicolumn{3}{|c|}{ Desvio Padrão (MPa) } & \multicolumn{9}{|c|}{0,0391} \\
\hline \multicolumn{3}{|c|}{ C.V. (\%) } & \multicolumn{9}{|c|}{36,04} \\
\hline
\end{tabular}

Após a execução do ensaio foram aferidos quatro diferentes formas de rompimento, duas do tipo coesiva (ruptura na argamassa e ruptura no chapisco) e duas do tipo adesiva (ruptura na interface chapisco/argamassa e interface chapisco/substrato). Com a média dos resultados obtidos notou-se que o aditivo adesivo proporcionou uma melhora de 14\% no desempenho da aderência da argamassa. 


\section{CONCLUSÃO}

Os resultados obtidos através dos ensaios realizados, foi possível concluir que o uso do aditivo adesivo influencia melhoras na argamassa tanto no estado fresco como no estado endurecido. Foi verificado que a argamassa com aditivo obteve $2 \%$ de melhora na consistência em relação à convencional. $\mathrm{O}$ aditivo apresentou um bom desempenho no quesito da permeabilidade e uma melhora significativa, em torno de $14 \%$, na aderência da argamassa com a presença do aditivo. Fazendo uma ressalva que o uso do chapisco influenciou na aderência do substrato com a argamassa.

Percebe-se, que as médias obtidas através do ensaio de resistência de aderência à tração não atenderam as exigências da NBR 13528 (ABNT, 2010). Um aspecto que pode ter influenciado nos resultados foi o tipo de aplicação da argamassa. Outros fatores que influenciam no resultado final da aderência é a escolha dos materiais e do traço empregado, a absorção de água das argamassas, a correta aplicação do aditivo adesivo na argamassa ainda em seu estado fresco, o correto modo de preparo da superfície aliado a uma limpeza minuciosa da base colante deixando a mesma livre de intempéries e oleosidades, na introdução das forças que serão aplicadas no revestimento de argamassa e consequentemente na extensão do contato entre revestimento e substrato. Sobre a consistencia dos dados avalia-se que todos possuíram CV superior a 30\%, de acordo com Garcia (1989) resultados como este são considerados de elevada dispersão. Apesar dos resultados não se mostrarem confiáveis, destaca-se que no estudo sobre a aderência de argamassas é comum encontrar pesquisas com coeficiente de variação acima de $30 \%$ para uma mesma situação de ensaio, de acordo com Costa e Carasek (2009) isto ocorre devido a: influência do procedimento de corte, geometria e dimensão dos corpos de prova, camada de cola, excentricidade da pastilha, tipo de equipamento e taxa de carregamento.

\section{REFERÊNCIAS}

ASSOCIAÇÃO BRASILEIRA DE NORMAS TÉCNICAS. NBR 7200: Execução de revestimentos de paredes e tetos de argamassas inorgânicas - Procedimento. Rio de Janeiro, 1998.

NBR13276: Argamassa para assentamento e revestimento de paredes e tetos - Preparo da mistura e determinação do índice de consistência. Rio de Janeiro, 2005.

NBR13278: Argamassa para assentamento de paredes e revestimento de paredes e tetos - Determinação da densidade de massa e do teor de ar incorporado. Rio de Janeiro, 2005.

. NBR 13528: Revestimento de paredes e tetos de argamassas inorgânicas - Determinação da resistência de aderência à tração. Rio de Janeiro, 2010.

NBR 13749: Revestimento de paredes e tetos de argamassas inorgânicas: especificação. Rio de Janeiro, 2013.

CARASEK, H. Aderência de Argamassas à base de cimento Portland a substratos porosos. Tese de Doutorado, Escola politécnica da Universidade de São Paulo, São Paulo, 1996.

CARAseK, H. Argamassas. IBRACON. Materiais de Construção Civil e Princípios de Ciência e Engenharia de Materiais. São Paulo, 2007.

CENTRE SCIENTIFIQUE ET TECHNIQUE DE LA CONSTRUCTION - CSTC. Hydrofuges de surface: choix et mise em oeuvre. Bruxelles. (Note D`Information Technique - NIT n. 140), 24 p., 1982.

COSTA, E. B. Ca.; CARASEK, H.. Influência dos parâmetros de ensaio na determinação da resistência de aderência de revestimentos de argamassa. Ambiente Construído, v. 9, n. 4, p. 17-35, 2009.

Garcia CH (1989) Tabelas para classificação do coeficiente de variação. Piracicaba, IPEF. 12p. (Circular técnica, 171).

GOMES, A. O.; NEVES, C. M. M. Proposta de método de dosagem racional de argamassas contendo argilominerais. Publicação ANTAC, 2002. 


\section{CВPAT 2020 \\ CONGRESSO BRASILEIRO DE PATOLOGIA DAS CONSTRUÇÕES \\ DE 15 A 17 DE ABRIL | FORTALEZA - CE \\ ISBN 978-65-86819-05-2}

lOPPI, P. R. Estudo da aderência das argamassas de revestimento em substratos de concreto. Florianópolis, 1995. 135f. Dissertação (Mestrado em Engenharia Civil) - Curso de Pós Graduação em Engenharia Civil, Universidade Federal de Santa Catarina.

SANTOS H. B. Ensaio de aderência das argamassas de revestimento. Dissertação de Pós-Graduação, Universidade Federal de Minas Gerais, Belo Horizonte, 2008.

ZANELATO, E. B. Influência do chapisco na resistência de aderência à tração de revestimentos de argamassa em blocos cerâmicos. Universidade Estadual do Norte Fluminense Darcy Ribeiro, Campos dos Goytacazes - RJ, 2015. 